\title{
Enaminones as intermediates in the synthesis of indolizidine alkaloids
}

\author{
Joseph P. Michael* and David Gravestock \\ Centre for Molecular Design, Department of Chemistry, University of the Witwatersrand, \\ Wits 2050, South Africa
}

\begin{abstract}
The use of vinylogous urethanes as pivotal intermediates in the synthesis of indolizidine alkaloids is illustrated with reference to indolizidines 209B (1) and 167B (2), two simple alkaloids isolated from dendrobatid frogs. Methodology is optimized for the diastereoselective synthesis of the racemic alkaloids, after which modifications leading towards the enantioselective synthesis of (-)-indolizidine 209B are presented.
\end{abstract}

\section{Introduction}

Nearly three hundred alkaloids belonging to about two dozen different structural classes have been partially or fully characterized from the skin secretions of amphibians (ref. 1). One of the largest of these classes is the indolizidine alkaloids, which occur in dendrobatid frogs from Central and South America as well as in some unrelated frogs and toads from locations as far apart as Australia and Madagascar. The most thoroughly explored amphibian indolizidines are the pumiliotoxins and allopumiliotoxins, but the more recently discovered 5-alkylindolizidines and 3,5- and 5,8-disubstituted indolizidines are now attracting increasing attention. Because of their extremely low natural abundance, there is still uncertainly about the relative and absolute stereostructure of many of these alkaloids, and this accounts for at least part of their popularity as synthetic targets. They are also simple enough to use as models for illustrating new synthetic methodologies. In their own right, however, they are potentially useful tools for neurophysiological applications because they are non-competitive blockers of sodium ion influx through nicotinic receptor channels both in muscle and in ganglia (ref. 2). Two simple indolizidime alkaloids, 209B and 167B, which have the tentative but widely accepted structures shown as 1 and 2 respectively, have provided us with an opportunity for investigating the stereochemical implications of a generalized synthetic strategy that we have been developing for several years (ref. 3).
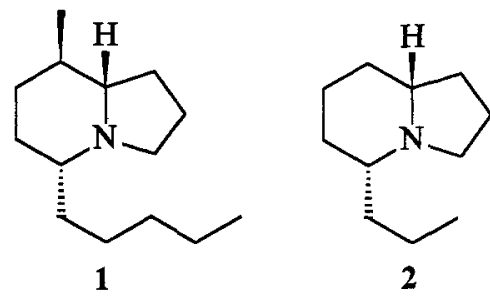

\section{Synthetic planning for indolizidine 209B}

In seven of the nine reported syntheses of indolizidine 209B, the bicyclic nucleus was assembled by making the $\mathrm{C} 3-\mathrm{N}$ bond, i.e., by cyclization on to nitrogen of a suitably functionalized 2-substituted piperidine derivative, as shown in the simplified disconnection overleaf (refs. 4 - 6). Our own approach to the indolizidine core involves an unconventional disconnection at the $\mathrm{C} 7-\mathrm{C} 8$ bond. The reason for this analysis becomes apparent once the synthon 3 is replaced by the synthetic equivalent 4 . The highlighted portion of this compound is an acylated enamine or "enaminone" (in this case, a vinylogous urethane), a structural unit that forms the focus of our alkaloid synthesis programme because of its ready accessibility, comparative stability, and - most importantly versatile reactivity. 


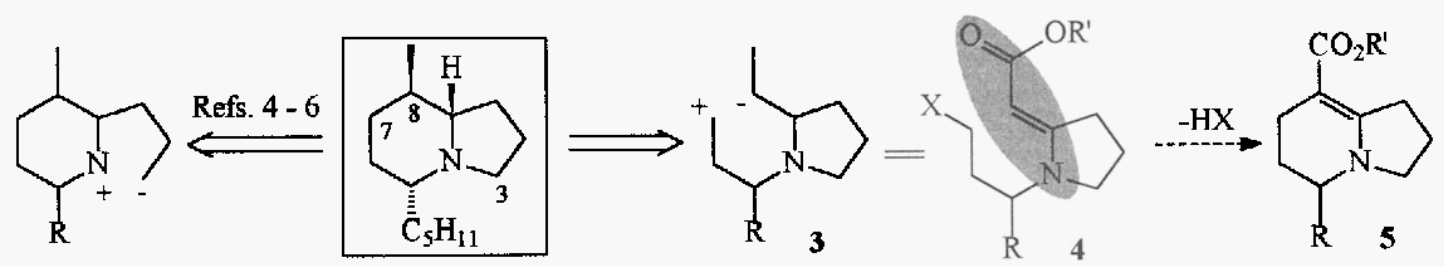

Our fascination with vinylogous urethanes and their relatives $\left(\mathrm{CO}_{2} \mathrm{R}\right.$ replaced by $\mathrm{COR}, \mathrm{CN}, \mathrm{NO}_{2}, \mathrm{SO}_{2} \mathrm{Ar}$, etc) stems from their manifold reactivity both as ambident nucleophiles and as ambident electrophiles, as shown in the box below. The expected "enamine" nucleophilicity at $\mathrm{N}$ and $\mathrm{C}$ can be extended to the carbonyl oxygen atom by conjugation (top row). In addition, deprotonation with strong bases can provide an additional nucleophilic site $\beta$ to $\mathrm{N}$ (bottom row, left). However, the systems also bave "enone" character, and hence may act as electrophiles at the carbon atoms $\alpha$ and $\gamma$ to $\mathrm{N}$. We exploit this nultiple reactivity by building the $\mathrm{N}-\mathrm{C}=\mathrm{C}-\mathrm{X}$ unit into systems containing additional nucleophilic or electrophilic sites that can serve as complementary reaction partners. In this way the enaminone functions as a scaffold for further annulation. The three examples shown below are taken from our published work, and illustrate ring closure by cycloacylation, cycloalkylation and cycloarylation as a prelude to the synthesis of ipalbidine (ref. 7), lamprolobine (ref. 8) and the mitosene ring system (ref. 9) respectively.
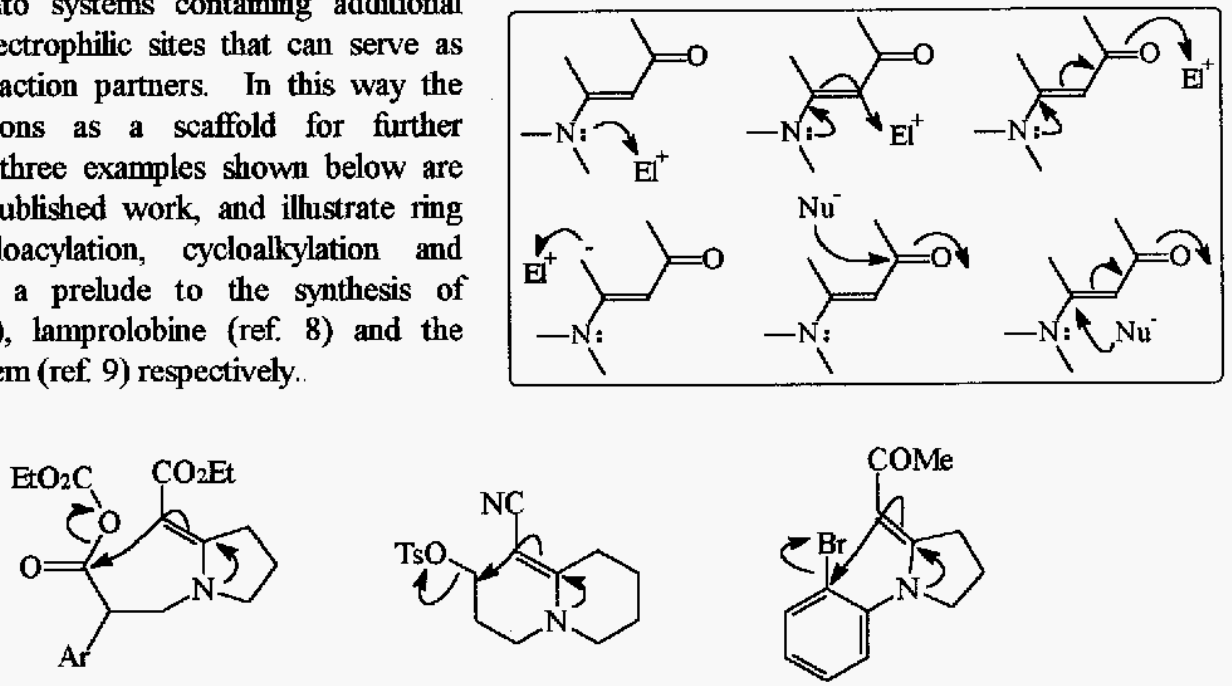

In the present work, a more challenging synthetic task occurs after cyclization to the unsaturated indolizidine nucleus 5 has been achieved. Once the $\mathrm{C}=\mathrm{C}$ bond has fulfilled its primary function as a component of the enamine system, it must be reduced diastereoselectively in order to set up the correct relative stereochemistry of the target alkaloids. Only when the basic methodology is in place will the further challenge of modifying the approach to ensure enantioselectivity as well as diastereoselectivity be worth confronting. In the following discussion, our successful route to racemic indolizidines $209 \mathrm{~B}$ and $167 \mathrm{~B}$ will be presented before the enantioselective modifications developed for the synthesis of (-)-indolizidine $209 \mathrm{~B}$ are described.

\section{Synthesis of ( \pm --indolizidine 209B and a new diastereoisomer of $209 \mathrm{~B}$}

Ethyl oct-2-enoate was prepared in $88 \%$ yield as a $9: 1$ mixture of $(E)$ and $(Z)$ isomers by Wittig reaction between hexanal and ethoxycarbonyltriphenytphosphorane. On treatment at room temperature with a catalytic amount of sodium hydroxide in tetrahydrofuran, the ester underwent conjugate addition with pyrrolidine-2thione to give thiolactam 6 ( $74 \%$ ). Alkylation on sulfur with ethyl bromoacetate followed by Eschenmoser sulfide contraction (ref. 10) upon treatment with triphenylphosphine and triethylamine in acetonitrile afforded the vinylogous urethane $7(85 \%)$. In order to construct the indolizidine nucleus by the sort of cycloalkylation process shown in the transformation $4 \rightarrow 5$, it was necessary to reduce the saturated ester group while leaving the unsaturated ester of the vimylogous urethane untouched. Fortunately, the latter functional group resists reduction with lithium aluminium hydride, attesting to the pronounced stability that extended conjugation imparts to the system. The desired alcohol 8 was isolated in $91 \%$ yield. The scene was thus set for the critical cyclization, which required conversion of the primary alcohol into a better leaving group. This troublesome step was eventually accomplished via the corresponding bromide, prepared in situ by treatment of 8 with carbon tetrabromide and triphenylphosphine in the presence of triethylamine. Simply refluxing the reaction mixture in acetonitrile was sufficient to bring about ring closure to the pivotal indolizidine $9(85 \%)$. 


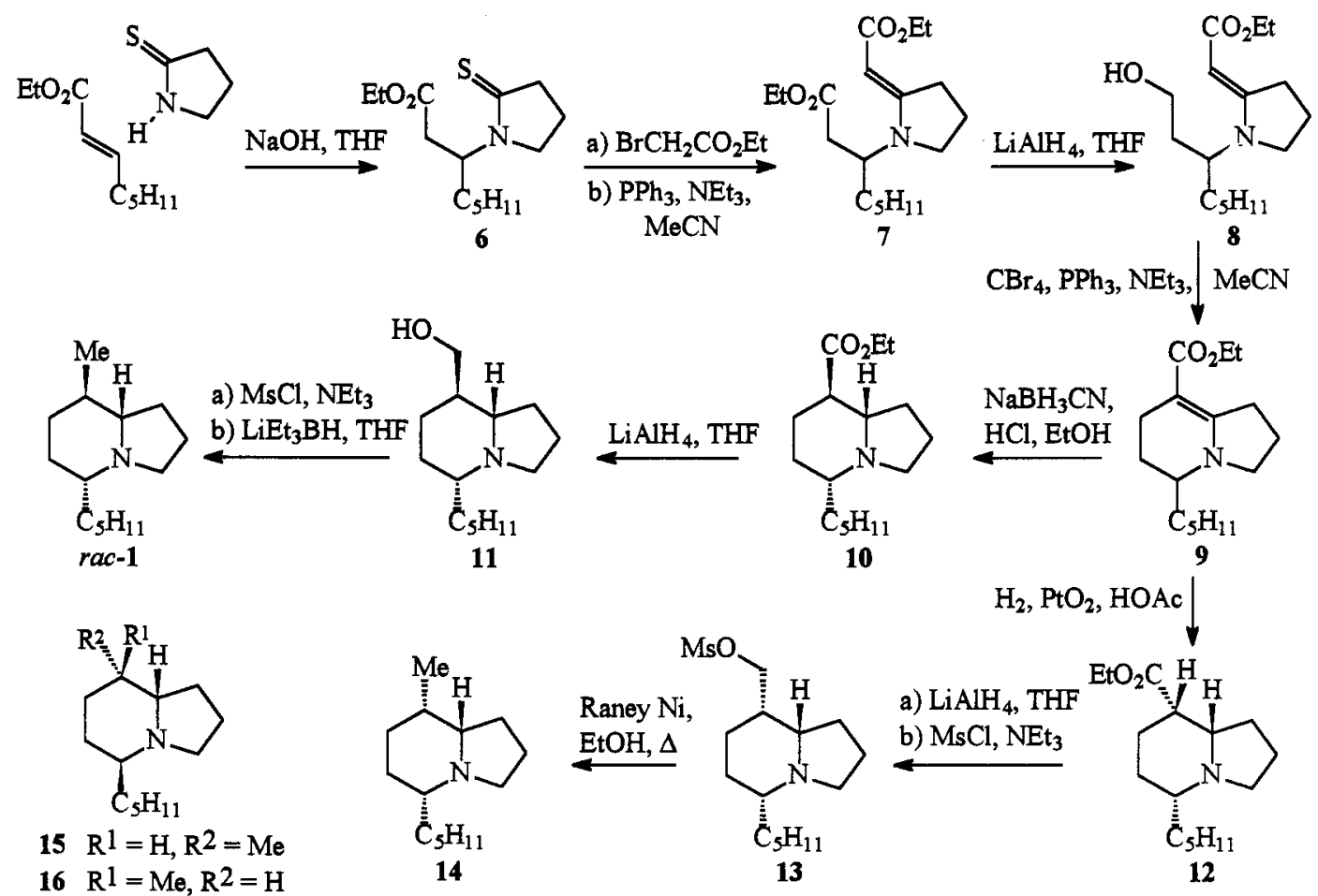

The most demanding task in our synthetic plan still lay ahead. By reducing the $\mathrm{C}=\mathrm{C}$ bond of 9 we are introducing two new stereogenic centres whose stereochemistry has to be controlled not only in relation to each other, but also in relation to the more remote stereogenic centre already present at $\mathrm{C} 5$. We reasoned that conformational effects in the bicyclic system would dictate the transition state that develops during the reduction. The incipient chair conformation of the six-membered ring should result in an equatorial preference for the pentyl side chain, which in turn should bias the approach of the reductant towards the distal face of the $\mathrm{C}=\mathrm{C}$ bond. Furthermore, protonating the vinylogous urethane will give an iminium ion whose reduction should be subject to stereoelectronic control according to the principles adumbrated by Stevens (ref. 11), which favour a transition state in which maximum orbital overlap between the approaching nucleophile and the developing lone pair on nitrogen is maintained. In the event, the observed diastereoselectivity on treatment of 9 with sodium cyanoborohydride at $\mathrm{pH} 4$ was disappointing, since the desired compound 10 (33\%) was accompanied by two diastereoisomers $(14 \%+13 \%)$. The structure of 10 was confirmed by reduction with lithium aluminium hydride to the alcohol $11(92 \%)$, the spectroscopic data for which matched those reported by Holmes (ref. 4) and Jefford (ref. 12). Holmes has transformed the (-)-enantiomer of 11 into the target alkaloid 1 by reducing the mesylate derivative with lithium triethylborohydride. We repeated these steps with the racemic alcohol 11 to give a volatile product ( $40 \%$ over two steps) whose NMR spectra were identical with those published for 1 (refs. 4 - 6). The overall yield in this eight-step preparation of ( \pm )- 1 from pyrrolidine-2-thione was $5.9 \%$.

A far more diastereoselective reduction of bicyclic vinylogous urethane 9 was achieved with hydrogen and a platinum catalyst in acetic acid. Hydrogen was delivered in the expected cis fashion on to the less hindered face of the $\mathrm{C}=\mathrm{C}$ bond to afford the new ester $12(71 \%)$. Compound 10 was a minor product of this reduction $(<6 \%)$. Further reduction of 12 with lithium ahminium hydride followed by mesylation yielded compound 13 (88\%, two steps). Since we found that demesylations with lithium triethylborohydride were erratic, we investigated alternative reagents for this defunctionalization. Somewhat surprisingly, we were able to produce the 8-methyl-5-pentylindolizidine 14 in $65 \%$ yield by heating 13 with Raney nickel under reflux in ethanol. Compound 14 is a hitherto unknown diastereoisomer of indolizidine 209B; its NMR spectra are distinctly different from those of 209B and the only other reported diastereoisomer, which appears in the literature as 15 (ref. 5) but has subsequently been revised to 16 (ref. 13). Pohiaszek and Belmont have reported valuable ${ }^{1} \mathrm{H}^{\prime}$ and ${ }^{13} \mathrm{C}$ NMR data for all four diastereoisomers of indolizidine 205A, which differs from $209 \mathrm{~B}$ only in having a pent-4-ynyl side chain (ref. 14). The chemical shifts we found for the indolizidine nucleus of 14 are in remarkable agreement $( \pm 0.3 \mathrm{ppm})$ with those of the analogous isomer of $205 \mathrm{~A}$. 


\section{Synthesis of indolizidine 167B}

In the above synthesis of indolizidine 209B, the ester group of vinylogous urethane 9 serves not only to temper the reactivity of the enaminone system, but also functions as the source of the target alkaloid's 8-methyl group. Adapting the methodology for the synthesis of indolizidine 167B requires that this ester group be dispensed with once the vinylogous urethane unit has served its purpose. If the indolizidine nucleus were to be assembled by cycloacylation rather than cycloalkylation, the ester would become a component of a $\beta$-keto ester system, and thus readily removed by hydrolysis and decarboxylation. Accordingly, the previous synthesis was repeated as far as the sulfide contraction step, but with the pentyl chain replaced by propyl (46\%, two steps). Hydrolysis of the saturated ester group of product $\mathbf{1 7}$ was achieved with one equivalent of sodium hydroxide in refluxing aqueous solution. Once again, this reaction attests to the unusual stability of the vinylogous urethane unit, which emerged unscathed. Cyclization was achieved via a mixed anhydride made in situ from the thoroughly dried carboxylate salt 18 by treatment with acetic anhydride and potassium carbonate in dry acetonitrile $(89 \%$ over two steps). Reftuxing the bicyclic compound 19 with aqueous potassium hydroxide solution followed by concentrated hydrochloric acid then afforded the vinylogous amide $20(91 \%)$. Our prior experience shows that the $\mathrm{C}=\mathrm{C}$ double bond in such compounds can be chemoselectively reduced with lithium aluminium hydride as long as one avoids an excess of the reagent (ref 3). In this case, 20 was reduced to the simple indolizidinone 21 (56\%), which was apparently free of the other possible diastereoisomer. The final defunctionalization was achieved via propylene dithioacetal $22\left[\mathrm{HS}\left(\mathrm{CH}_{2}\right)_{3} \mathrm{SH}, \mathrm{CF}_{3} \mathrm{CO}_{2} \mathrm{H}, \mathrm{BF}_{3} . \mathrm{OEt}_{2} ; 94 \%\right]$, which underwent hydrogenolysis to ( \pm -indolizidine 167B (2) with Raney nickel in boiling ethanol (37\%). The low yield can be ascribed in part to the volatility of the alkaloid. The NMR spectra of the product were in perfect agreement with those reported in the literature (refs. 15, 16), and the ${ }^{13} \mathrm{C}$ NMR spectrum in particular was quite different from that reported for the altemative diastereoisomer (ref. 15).

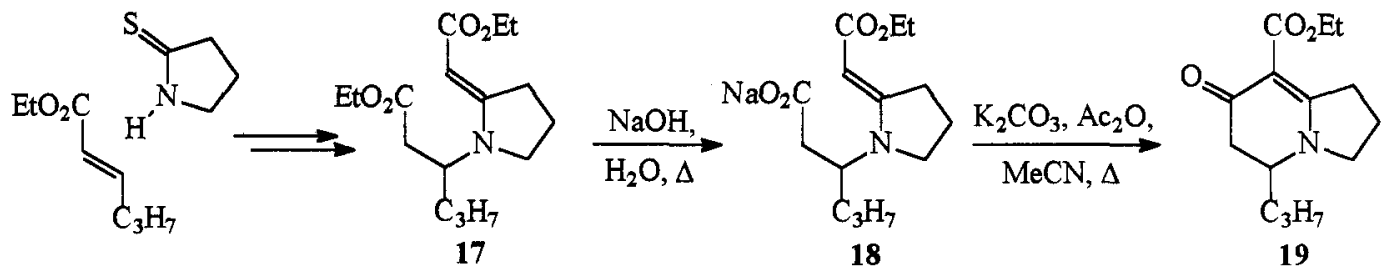

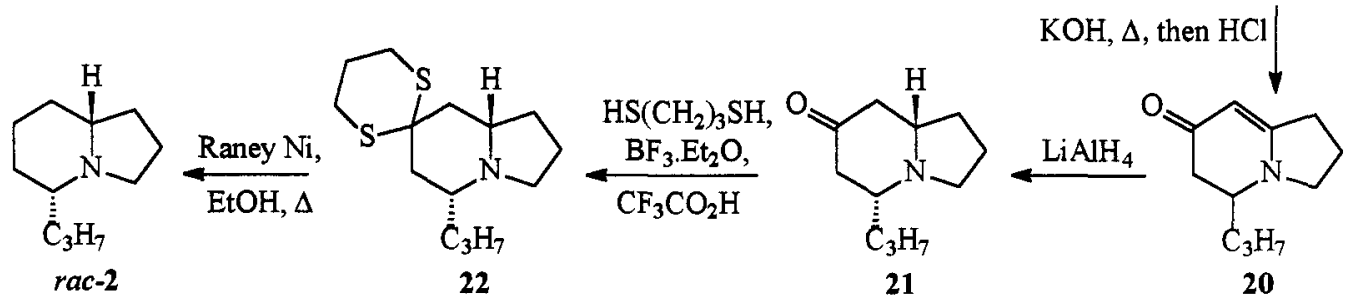

\section{Enantioselective modifications: synthesis of (-)-indolizidine 209B}

Having established the viability of our synthetic approach for making indolizidines, we turned our attention to enantioselective modifications of our methodology. The step needing attention occurs at the beginning of the synthetic sequence, when the conjugate addition of pyrrolidine-2-thione to ethyl alk-2-enoate results in the creation of the first stereogenic centre. In principle, a diastereofacially biased addition to a geometrically pure alkenoate as shown overleaf should result in an adduct enriched in one enantiomer. The simplest way to introduce the necessary bias would be to replace the ethyl ester by a suitable chiral auxiliary, when the addition process should give a potentially separable mixture of diastereoisomers. We investigated this strategy with several well-known auxiliaries, using the Horner-Wadsworth-Emmons reaction between hexanal and appropriate diethoxyphosphorylacetates to prepare oct-2-enoates that consisted (within the limits of NMR detection) entirely of the $(E)$ isomer ( $>99: 1)$. The diastereoselectivity in these additions proved to be modest, reaching at most about 2:1 with the 8-phenylmenthyl ester 23 . The trouble arose because the conjugate addition turned out to be an equilibrium process that almost certainly proceeded by S-alkylation before the more stable $\mathrm{N}$-alkyl product formed. We were never able to achieve a kinetically controlled conjugate addition with pyrrolidine-2-thione as nucleophile. 


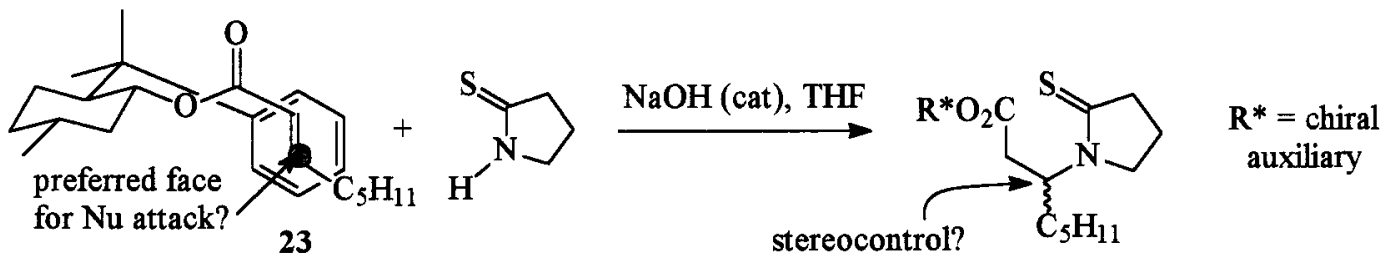

We clearly needed to find a more cooperative nucleophile than pyrrolidine-2-thione if we were to achieve the critical diastereofacial selectivity required for a successful enantioselective route to 209B. Looked at in a different light, the synthetic feat we have to achieve is the enantioselective synthesis of a $\beta$-amino acid derivative, around which has built up a vast body of literature (ref. 17). Amongst several protocols involving the conjugate addition of nitrogen nucleophiles to unsaturated esters, recent methodology devised by Davies and Ichihara (ref. 18) seems tailor-made for our purposes. This comparatively general route involves the conjugate addition of lithium $N$-benzyl- $N$-[(1R)-1-phenylethyl]amide or its enantiomer to $t$-butyl $(E)$-alk-2-enoates to give the desired adducts in excellent chemical yields, reproducibly high diastereoselectivities ( $>95 \%$ d.e.), and with a predictable stereochemical outcome (ref. 19). Hydrogenolytic removal of the benzyl groups ( $7 \mathrm{~atm} \mathrm{H}_{2}, 10 \% \mathrm{Pd}-\mathrm{C}$, acetic acid) then yields enantiomerically pure $\beta$-amino esters.

We were delighted when the conjugate addition of lithium $N$-benzyl- $N-[(1 R)-1$-phenylethyl] amide to $t$-butyl $(E)$ oct-2-enoate yielded a diastereomerically pure adduct 24 in $90 \%$ yield. Analogies with closely related examples reported by Davies (ref. 20) suggested that this adduct should have $(R)$ absolute configuration at the newly created stereogenic centre. Independent confirmation of the absolute stereostructure was obtained by means of $X$-ray crystallography on the hydrobromide salt of the corresponding carboxylic acid (ref. 21). Interestingly, the results confirmed not only the $(R)$ configuration at both stereogenic carbon atoms, but also showed that the protonated nitrogen atom has $(S)$ configuration. Debenzylation under Davies conditions gave the free amino ester $25(92 \%)$, thereby opening the way for the construction of the lactam ring in 26 , which was accomplished with 4-chlorobutyryl chloride followed by cyclization with potassium $t$-butoxide (82\%). The corresponding thione 27 was prepared from 26 with Lawesson's reagent in boiling toluene (89\%). Sulfide contraction completed the synthesis of $(R)$-vinylogous urethane 28 (94\%). An alternative one-pot route to 28 involving

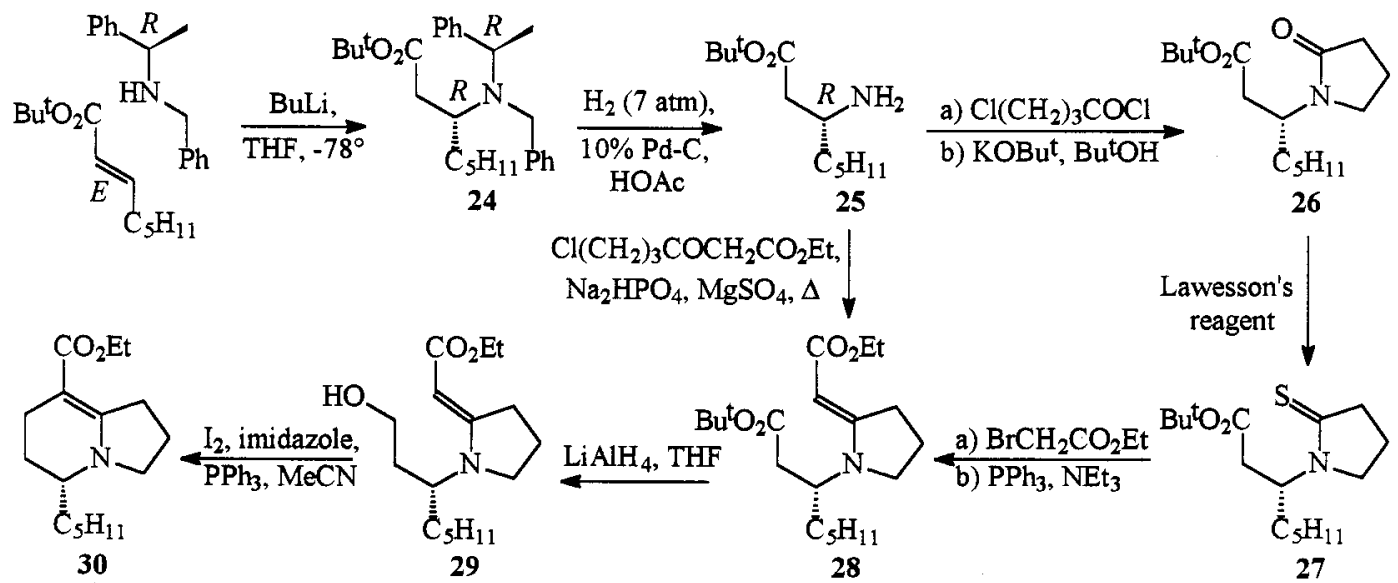

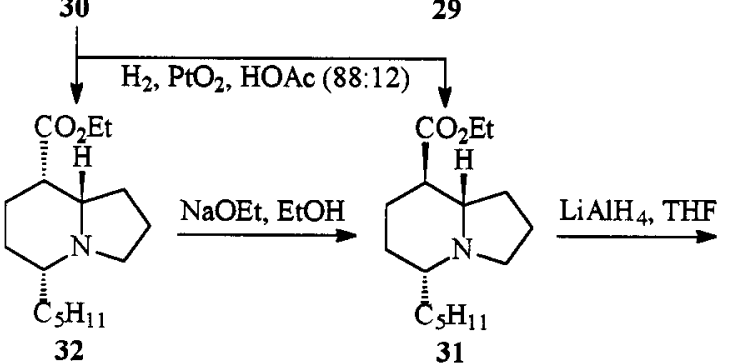

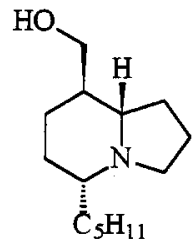

33<smiles>[Y4]C1CCC([Z11])C2(CCCN2)C1CCC</smiles>

$(-)-1$ 
treatment of amine 25 with ethyl 6-chloro-3-oxohexanoate was shorter but less efficient (49\%; cf. 69\% for the four-step process). Reduction of 28 with lithium aluminium hydride yielded the alcohol 29 (88\%), at which stage the synthesis converges with that described above for racemic indolizidine 209B. However, we introduced several minor changes to improve the overall process. Firstly, when cycloalkylation of 29 via the bromide proved erratic, we were consistently successful with the corresponding iodide $(81 \%)$. Secondly, reduction of the $\mathrm{C}=\mathrm{C}$ bond of the bicyclic vinylogous urethane 30 with sodium cyanoborohydride at $\mathrm{pH} 4$ was again insufficiently diastereoselective for our needs, since it afforded 31 in only $36 \%$ yield. By contrast, catalytic hydrogenation ( $1 \mathrm{~atm} \mathrm{H}_{2}, \mathrm{PtO}_{2}$, acetic acid) resulted in the highly diastereoselective formation of 32 together with $31(88: 12,85 \%)$. The axial ester group of 32 proved amenable to epimerization when the compound was heated with a catalytic quantity of sodium ethoxide in ethanol. Conversion into the desired equatorial isomer 31 was essentially complete (glc analysis), although its recovery after column chromatography was not especially good (40\%). Finally, reduction of 31 with lithium ahuminium hydride yielded alcohol $33(94 \%)$, thereby completing a formal synthesis of (-)-indolizidine 209B (1). The spectroscopic data were in excellent agreement with those reported in the literature, as was the optical rotation, $[\alpha]_{D}-93.1(c 0.58, \mathrm{MeOH})[c f .-93.3(c 0.58$, $\mathrm{MeOH})$, ref $4 ;-93.6$ (c 0.51, MeOH), ref 12]. Holmes's conversion of (-)-31 into (-)-indolizidine 209B (1) via the corresponding mesylate (ref 4 ) has been referred to previously.

\section{Acknowledgements}

We thank the Foundation for Research Development, Pretoria, and the University of the Witwatersrand for funding this research.

\section{References}

1. J.W. Daly, H.M. Garraffo and T.F. Spande, Alkaloids (N.Y.) 43, 185 (1993).

2. J.W. Daly, Y. Nishizawa, W.L. Padgett, T. Tokuyama, A.L. Smith, A.B. Holmes, C. Kibayashi and R.S. Aronstam, Neurochem. Res. 16, 1213 (1991).

3. J.P. Michael and A.S. Parsons, Tetrahedron 52, 2199 (1996); and references cited therein.

4. A.B. Holmes, A.L. Smith, S.F. Williams, L.R. Hughes, Z. Lidert and C. Swithenbank, J. Org. Chem. 56, 1393 (1991).

5. D. Gnecco, C. Marazano and B.C. Das, J. Chem. Soc., Chem. Commun. 625 (1991).

6. For other syntheses of indolizidine 209B using an N-C3 disconnection, see the following: D.L. Comins and E. Zeller, Tetrahedron Lett. 32, 5889 (1991); Y. Shishido and C. Kibayashi, J. Org. Chem. 57, 2876 (1992); A. Satake and I. Shimizu, Tetrahedron: Asymmetry 4, 1405 (1993); T. Momose and N. Toyooka, J. Org. Chem. 59, 943 (1993); J. Ahman and P. Somfai, Tetrahedron 51, 9747 (1995). For syntheses of indolizidine 209B using alternative disconnections see J. Aubé, P.S. Rafferty and G.L. Milligan, Heterocycles 35, 1141 (1993); and ref. 12. A.S. Howard, G.C. Gerrans and J.P. Michael, J. Org. Chem. 45, 1713 (1981).

J.P. Michael and C.M. Jungmann, Tetrahedron 48, 10211 (1992).

J.P. Michael, S.S.-F. Chang and C. Wilson, Tetrahedron Lett. 34, 8365 (1993).

10. K. Shiosaki. In Comprehensive Organic Synthesis (B.M. Trost, ed.), Vol, 2, pp. 865-892. Pergamon Press, Oxford (1991).

11. R. Stevens, Acc. Chem. Res. 17, 289 (1984).

12. C.W. Jefford, K. Sienkiewicz and S.R. Thornton, Helv. Chim. Acta 78, 1511 (1995).

13. Dr C. Marazano, CNRS, Gif-sur-Yvette, personal communication. We are grateful to Dr Marazano for his kind assistance with a number of our queries.

14. R.P. Polniaszek and S.E. Belmont, J. Org. Chem. 56, 4868 (1991).

15. R.P. Polniaszek and S.E. Belmont, J. Org. Chem. 55, 4688 (1990).

16. For other syntheses of indolizidine $167 \mathrm{~B}$, see ref. 4 and the following: C.W. Jefford. Q. Tang and A. Zaslona, J. Am. Chem. Soc. 113, 3513 (1991); E. Zeller, H. Sajus and D. Grierson, Synlett 44 (1991); A. Fleurant, J.P. Célérier and G. Lhommet, Tetrahedron: Asymmetry 3, 695 (1992); W.H. Pearson, R Walavalkar, J.M. Schkeryantz, W.-K. Fang and J.D. Blickensdorf, J. Am. Chem. Soc. 115, 10183 (1993); C.W. Jefford and J.B. Wang, Tetrahedron Lett. 34, 3119 (1993); A. Fleurant, C. Saliou, J.P. Célérier, N. Platzer, T.V. Moc and G. Lhommet, J. Heterocyclic Chem. 32, 255 (1995); H. Takahata, H. Bandoh and T. Momose, Heterocycles 41, 1797 (1995); E. Lee, K.S. Li and J. Lim, Tetrahedron Lett. 37, 1445 (1996).

17. D.C. Cole, Tetrahedron 50, 9517 (1994).

18. S.G. Davies and O. Ichihara, Tetrahedron: Asymmetry 2, 183 (1991).

19. J.F. Costello, S.G. Davies and O. Ichihara, Tetrahedron: Asymmetry 5, 1999 (1994).

20. M.E. Bunnage, A. Burke, S.G. Davies and C.J. Goodwin, Tetrahedron: Asymmetry 6, 165 (1995).

21. J.C.A. Boeyens and C.F. Broli, unpublished results, University of the Witwaterstand (1995). 